\title{
Power System Stability Enhancement using SVC with Modified PSO Tuned PID Controller
}

\author{
NizarHadi Abbas \\ Electrical Engineering Department \\ College of Engineering, University \\ of Baghdad, Iraq.
}

\author{
Ruba AL-MulaHumadi \\ Mechanical Engineering Dept. \\ College of Engineering, University \\ of Baghdad, Iraq.
}

\begin{abstract}
A modified particle swarm optimization (MPSO) algorithm is utilized in this paper to find out the optimum design of Proportional-Integral-Derivative (PID) controller parameters that improves the stability of a Single-Machine Infinite-Bus (SMIB) system with Static VAR Compensator (SVC). The performance of the power system under different loading conditions is investigated by using three tuning methods. Namely, Ziegler-Nichols-PID, PSO-PID, and MPSO-PID.The simulation results show that the proposed MPSO-PID controlling technique has improved the system response as compared to the two other techniques, that because it gives minimum rise time and minimum settling time with no overshoot and approximately zero steady-state error. All simulations are carried out in MATLAB R2010a software environment.
\end{abstract}

\section{Keywords}

SMIB Power System, SVC, PID Controller, Ziegler-Nichols Tuning Method, PSO Algorithm, MPSO Algorithm, Power System Stability.

\section{INTRODUCTION}

The stability of a power system is its ability to develop restoring forces equal to or greater than the physical disturbing forces to return to an equilibrium state [1]. Nowadays, the stability improvement issue is gradually becoming popular and play as a critical role in power system. There are various approaches available in literature for enhancing the power system stability, like real power, reactive power, voltage level, and machine oscillation damping controls, which are taken a great interest from numerous researchers to get better performance.

Rahman, et al. [2], presented SVC (Static VAR Compensator) controlled externally by a PID controller for better voltage stability. The parameters of the PID controller were selected by using Ziegler-Nichols tuning method. The authors here examined their system with both single phase and three phase faults. Their network was simulated in three steps, without SVC, With SVC \& with PID controlled SVC. They found that the system had less time and less damping with PID controller as compared with the case of without controller. The authors here mentioned other FACTS (Flexible AC Transmission Systems) devices as SSSC (Synchronous Static Compensator), STATCOM (Static Synchronous Compensator), and UPFC (Unified Power Flow Controller) whose controllers can be controlled externally by using different kinds of controllers to improve power system stability.

Rahman, et al. [3], proposed a new type of SVC, which is controlled externally by a PID controller to improve the stability. The authors introduced a Triple Integral Differential (TID) close loop tuning method to optimize the PID controller parameters. They applied their idea on a two-machine power system. Their simulated method was made in three steps: without SVC, with SVC but no externally controlled and with SVC controlled externally by TID -PID controller. Rahman, et al. examined the system for some types of faults, and they found that the system had better performance and became stable with SVC. The authors also suggested other FACTS devices such as STATCOM, UPFC, and SSSC that can be controlled externally by using different types of controllers tuned by Fuzzy logic (FL), Artificial neural network (ANN), Genetic algorithm (GA)...etc. for power system stability improvement.

Rahman, et al. [4], demonstrated a thyristor based SVC with dissimilar types of controllers such as POD, PI, PID \& generic controllers. They compared the system performance under different loading conditions and various types of faults to improve the stability of the multi-machine power system. The simulated results showed that with uncontrolled SVC, the system stability times become large while the controlled SVC gave less times, and the stability of the system improved. The authors concluded that the SVC with Generic controller is the most efficient for voltage stability \& machine damping oscillation, because of the shorter time as compared with the other types of controllers.

FACTS devices are used to improve power system stability. Different types of FACTS devices are available, such as Unified Power Flow Controller (UPFC), Synchronous Static Compensator (SSSC), Thyristor-Controlled Phase Shifter (TCPS), Thyristor-Controlled Series Capacitor (TCSC) , Static VAR Compensator (SVC), etc. were reviewed and discussed by A. K. Mohanty and A. K. Barik[5].

The SVC can be controlled using different type of controllers in order to achieve better performance. PID is a wellestablished type of controller and widely used in industrial plants. The method of determination of the PID controller parameters which is called tuning process is the main objective to attain optimal or suboptimal design for a closed loop control system. The classical PID controllers were tuned initially by J. G. Ziegler and N. B. Nichols [6].

Recently, a number of advanced evolutionary algorithms namely: Particle Swarm Optimization (PSO), Ant Colony Optimization (ACO) and ArtificialImmune System (AIS) have been widely used by researchers to solve the optimization problems in the electrical engineering field. PSO is an extremely simple, effective algorithm and can reach the global optima within a shorter time period [7]. Therefore, in this paper, PSO algorithm is chosen, to determine the optimum PID controller parameters that enhanced the power system stability. 
The objective of this research work is to study the stability enhancement issue of a single machine infinite bus power system based on the more recent and efficient swarm intelligence based optimization technique called modified particle swarm optimization in order to tune the PID controller parameters. The MPSO-PID tuning method is applied to the proposed SMIB with SVC model and then finds out its time-domain specifications such as rise time, maximum overshoot, settling time, and steady-state error, which are compared with the results obtained from PSO-PID and $\mathrm{ZN}-$ PID tuning methods.

The rest of the paper is organized as follows: Section 2, describes the modeling of SMIB with SVC. The concept of PID controller, PSO algorithm, and modified PSO algorithm are introduced in section 3. Section 4, presents the implementation details of ZN-PID, PSO-PID, and MPSO-PID tuning methods. A discussion for the simulated results and analysis is demonstrated in section 5. Some concluding remarks are highlighted in section 6 .

\section{Power System Model}

The mathematical model of a single machine with infinite bus (SMIB) with SVC has been described in this paper as shown in Fig. 1.

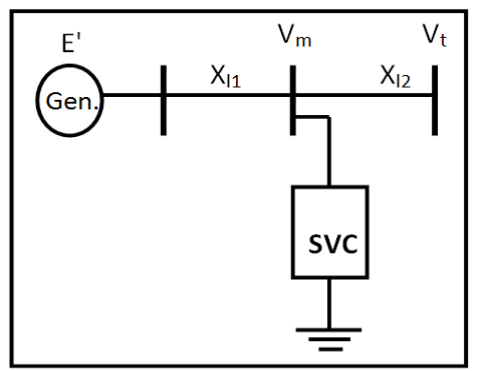

Fig 1: SMIB with SVC

SVC, is defined as a controlled susceptance (B), and it consists of a fixed capacitor (FC) connected in parallel with a thyristor controlled reactor (TCR).

SVC is used to inject reactive power to the system to increase the bus voltage to the desired level. And when the bus voltage exceeds the desired level, the SVC will absorb reactive power from the system in order to maintain the desired level. Fig. 2 shows the SVC model while Fig. 3 shows its characteristics $[3,8,9]$.Therefore, the mathematical model of SVC can be derived from V-I curve of SVC illustrated in Fig.3as follows:

$V=\left\{\begin{array}{cc}V_{\text {ref }}+I X_{S} & \quad: \text { In requaltion range } \\ \left(-B_{c \max }<B<B_{c \max }\right) \\ \frac{I}{B_{c \max }} \quad: \text { If SVC is fully capacitive } \\ \frac{I}{B_{l \max }} & \quad\left(B=B_{c \max }\right) \\ & \left(B=B_{l \max }\right)\end{array}\right\}$

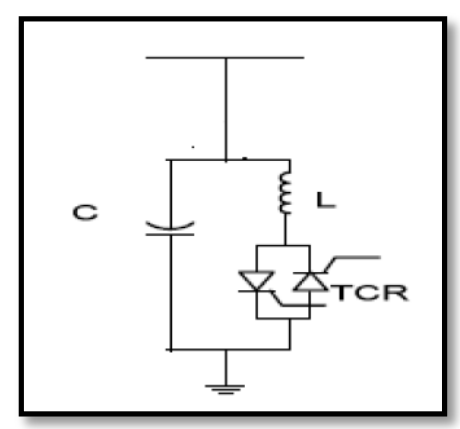

Fig2:The SVC Model

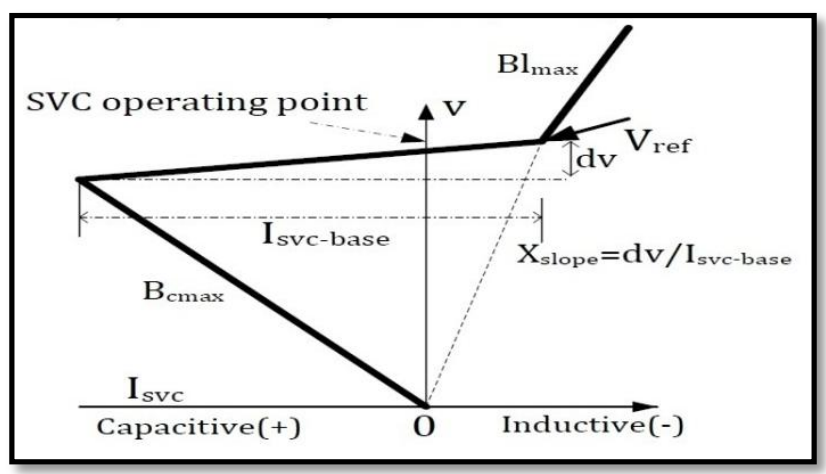

Fig 3: The V-I Characteristics of a SVC

The SVC magnitude is inductive admittance $B_{L}(\alpha)$ which is described as a function of the firing angle $\alpha[9]$ :

$$
B_{L}(\alpha)=\frac{2 \pi-2 \alpha+\sin 2 \alpha}{\pi x_{s}}
$$

where: $\pi / 2 \leq \alpha \leq \pi, x_{s}=v_{s}^{2} / Q_{L}$

$V_{s}:$ The bus bar voltage of the SVC.

$Q_{L}$ :MVA rating of reactor.

As stated above, the SVC is a combination of (TCR-FC), the effective shunt admittance will be [9]:

$$
\mathrm{B}_{\mathrm{s}}=\frac{1}{\mathrm{x}_{\mathrm{C}}}-\mathrm{B}_{\mathrm{L}}(\alpha)
$$

where $\mathrm{x}_{\mathrm{c}}$ is the capacitive reactance.

From Eq. (3), one can conclude that the SVC is used to improve the transient stability and power system oscillation damping and for that, SVC is placed at the midpoint of the transmission line.

The Mathematical model of SMIB system with SVC on the transmission line may be presented as [10]:

$$
\begin{gathered}
\dot{\delta}=\mathrm{w}-\mathrm{w}_{\mathrm{O}} \\
\dot{\mathrm{w}}=\frac{\mathrm{w}_{\mathrm{o}}}{\mathrm{H}}\left(\mathrm{B}_{\mathrm{m}}-\mathrm{P}_{\mathrm{e}}\right)-\frac{\mathrm{D}}{\mathrm{H}}\left(\mathrm{w}-\mathrm{w}_{\mathrm{o}}\right)(5) \\
\dot{B_{L}}=\frac{1}{\mathrm{~T}_{\mathrm{C}}}\left(-\mathrm{B}_{\mathrm{L}}+\mathrm{B}_{\mathrm{Lo}}+\mathrm{K}_{\mathrm{C}} \mathrm{u}_{\mathrm{B}}\right)(6) \\
P_{e}=\frac{\mathrm{E}^{\prime} \mathrm{v}_{\mathrm{C}}}{\mathrm{x}_{1}+\mathrm{x}_{2}+\mathrm{x}_{11} \mathrm{x}_{\mathrm{l}}\left(\mathrm{B}_{\mathrm{L}}-\mathrm{B}_{\mathrm{C}}\right)}
\end{gathered}
$$

And the mid bus voltage will be;

$$
V_{m}=\frac{\sqrt{\left(x_{12} E^{\prime} \cos \delta+x_{11} V_{t}\right)^{2}+x_{12} E^{\prime} \sin \delta^{2}}}{x_{1}+x_{2}+x_{11} x_{12}\left(B_{L}-B_{C}\right)}
$$

where,

$\delta: \quad \quad \quad$ Power angle of the generator in radian.

w: $\quad$ Rotor angular speed in radian.

$\mathrm{w}_{\mathrm{o}}$ : The synchronous machine speed $=2 \pi \mathrm{f}_{\mathrm{o}}$

$\mathrm{P}_{\mathrm{m}}$ : $\quad$ Mechanical input power in p.u.

$\mathrm{P}_{\mathrm{e}}$ : $\quad$ Electric power of generator in p.u. 


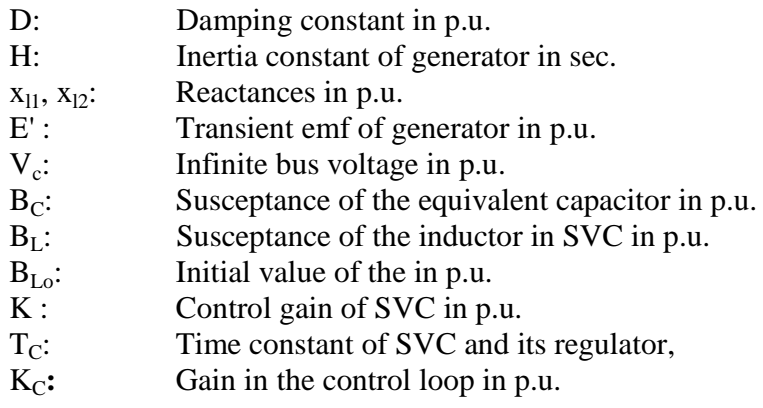

\section{Theoretical Basics}

\subsection{PID Controller}

PID controller is a combination of Proportional, Integral, and Derivative controllers. This is one of the most commonly used controllers in the industrial applications, because it offers simplest and yet most efficient solution in too many realworld control problems [11, 12].In proportional part of the controller, the actuating signal is reacted to the current error signal; the integral value has an output respond to sum of recent errors, and the derivative part determines the reaction based on the rate at which the error has been changing [13].

The actuating signal and the transfer function of PID controller can be given as $[14,15,16]$ :

$$
\begin{aligned}
& E_{a}(s)=K_{p} E(s)\left[1+s T_{d}+\frac{1}{s T_{i}}\right] \\
& G_{c}(s)=K_{p}+s K_{d}+\frac{K_{i}}{s}
\end{aligned}
$$

Where $K_{p}, K_{i}, K_{d}$ are the PID controller gain ,the time constants $T_{i}$ and $T_{d}$, called integral time (constant) and derivative time (constant), are sometimes used instead of the integral and derivative gains, $E(s)$ is the difference between the desired and obtained output, and $G_{c}(s)$ is the PID controller transfer function.

\subsection{Particle Swarm Optimization Algorithm}

Particle swarm optimization is a population based stochastic optimization method. This algorithm was inspired from the social behavioral pattern of organisms, such as Bird flocks, fish schools, and sheep herds where aggregated behaviors are met, producing powerful, collision-free, synchronized moves. In such systems, the behavior of each swarm member is based on simple inherent responses, although their collective outcome is rather complex from a macroscopic point of view. For example, the flight of a bird flock can be simulated with relative accuracy by simply maintaining a target distance between each bird and its immediate neighbors. This distance may depend on its size and desirable behavior. The swarms can also react to the predator by rapidly changing their form, breaking into smaller swarms and re-uniting, illustrating a remarkable ability to respond collectively to external stimuli in order to preserve personal integrity $[7,17]$.

The PSO algorithm consists of a number of particles that collectively move through the search space of the problem in order to find the global optima. Each particle is characterized by its position and fitness. Subsequently, the PSO algorithm updates the velocity vector for each particle then adds that velocity to the particle position. The velocity updates are influenced by both the best global solution associated with thehighest fitness ever found in the whole swarm, and the best local solution associated with the highest fitness in the present population. The velocity and position of each particle can be

$$
\begin{aligned}
& v_{i, m}^{t+1}=w v_{i, m}^{t}+c_{1} \operatorname{rand}_{1} *\left(P_{\text {best } i, m}-x_{i, m}^{t}\right) \\
& +c_{2} \operatorname{rand}_{2} *\left(g_{\text {best } i, m}-x_{i, m}^{t}\right) \\
& x_{i, m}^{t+1}=x_{i, m}^{t}+\Delta t v_{i, m}^{t+1}(12) \\
& \text { Where, } i=1,2, \ldots \ldots, \text { nand } m=1,2, \ldots \ldots ., d \text {; }
\end{aligned}
$$

$n:$

$d:$

$t:$

$v_{i, m}^{t}$

$v_{i, m}^{t+1}$ :

$w:$

$c_{1} \& c_{1}$

Number of particle in the swarm. Search space dimension.

Current iteration.

Current velocity of particle $i$ at iteration $t$.

Modified velocity of particle $i$.

Inertial weight factor.

. Cognitive and social acceleration factors respectively.

$\operatorname{rand}_{1} \& \operatorname{rand}_{1}$ : Random numbers uniformly distributed in the range $(0,1)$.

$x_{i, m}^{t}: \quad \quad$ Current position of particle $i$ at iteration $t$.

$x_{i, m}^{t+1}: \quad \quad$ Modified position of particle $i$.

$\Delta t: \quad$ Time step which is taken to be unity.

$P_{\text {best } i, m}: \quad$ The coordinate of the personal best position of the particle $i$ in $m^{\text {th }}$ dimension.

$g_{\text {best } i, m}$ : The coordinate of the global best position of the swarm in the $m^{\text {th }}$ dimension.

\section{Tuning of PID Controller 4.1PID Controller Tuning with Ziegler- Nichols Method}

There are two approaches for determining the PID controllers' parameters were presented by J. G. Ziegler and N. B. Nichols in 1942 [6]. The first design method (Z-N step response method) is based on the open-loop system which is characterized by the process time constant parameters $T$ and $L$, and the other method ( $\mathrm{Z}-\mathrm{N}$ frequency response method) is based on closed-loop that requires determination of the ultimate gain $K_{u}$ or critical gain $K_{c r}$ and the ultimate period $T_{u}$ or the oscillation period $P_{c r}$ [4].

In this paper, the Z-N frequency response method is used. In order to determine the PID controller parameters of the controlled plant, firstly, use the $\mathrm{P}$ controller only and its gain should be increased until the system reaches the critically stable region. Secondly, this value of gain is $K_{c r}$ and the corresponding period is $P_{c r}$. Additionally, these values can be found using root locus technique or any frequency domain plotting methods. Finally, the parameter of P, PI, and PID controller can be determined from $K_{c r}$ and $P_{c r}$ with the aid of the tuning formula illustrated in Table 1.

Table1.Ziegler-Nichols closed loop tuning parameter

\begin{tabular}{|l|c|c|c|}
\hline \multirow{2}{*}{$\begin{array}{c}\text { Controller } \\
\text { Type }\end{array}$} & \multicolumn{3}{|c|}{ Parameters } \\
\cline { 2 - 4 } & $\boldsymbol{K}_{\boldsymbol{p}}$ & $\boldsymbol{T}_{\boldsymbol{i}}$ & $\boldsymbol{T}_{\boldsymbol{d}}$ \\
\hline $\mathrm{P}$ & $0.5 K_{c r}$ & $\infty$ & 0 \\
\hline $\mathrm{PI}$ & $0.45 K_{c r}$ & $0.8 P_{c r}$ & 0 \\
\hline $\mathrm{PID}$ & $0.6 K_{c r}$ & $0.5 P_{c r}$ & $0.125 P_{c r}$ \\
\hline
\end{tabular}




\subsection{PID Controller Tuning with PSO Algorithm}

\section{A. Proposed PSO-PID}

The implementation of SVC controller in the SMIB system will enhance the system stability through selecting the optimal PID controller parameters of SVC using PSO-PID tuning method. Fig.4 shows the block diagram of proposed PSO/MPSO-PID controller for the SMIB system with SVC.

In the proposed PSO/MPSO-PID controlling method each particle contains three members $K_{p}, K_{i}, \& K_{d}$. In other words the problem search space has three dimensions and each particle in the population must fly in a three dimensional space. Fig. 5 illustrates the flowchart of implementing autotuning method for PID controller using PSO/MPSO algorithm to tune the PID Controller and collect the optimal parameters values.

The initial values for velocity vector and position vector in the first iteration can be taken as follows:

$$
\left.\begin{array}{l}
x_{i, 1}^{0}=K_{p}^{\text {min }}+\left(K_{p}^{\text {max }}-K_{p}^{\text {min }}\right) * \operatorname{rand}_{i, 1}(0,1) \\
x_{i, 2}^{0}=K_{i}^{\text {min }}+\left(K_{i}^{\text {max }}-K_{i}^{\text {min }}\right) * \operatorname{rand}_{i, 2}(0,1) \\
x_{i, 3}^{0}=K_{d}^{\text {min }}+\left(K_{d}^{\text {max }}-K_{d}^{\text {min }}\right) * \operatorname{rand}_{i, 3}(0,1)
\end{array}\right\}
$$

The values of $c_{1}$ and $c_{2}$ in Eq. (11) can affect the search ability of PSO by biasing the particles to the new best positions and the powerful values were set to be 1.494 as recommended in Clerc's PSO [18]. Another important factor that should initiate its value is thee inertial weight, $w$ which is involved in the Eq. (11) to make a balancing between global and local search capability and in order to get guaranteed convergence is set to 0.729 as recommended in Clerc's PSO [18].

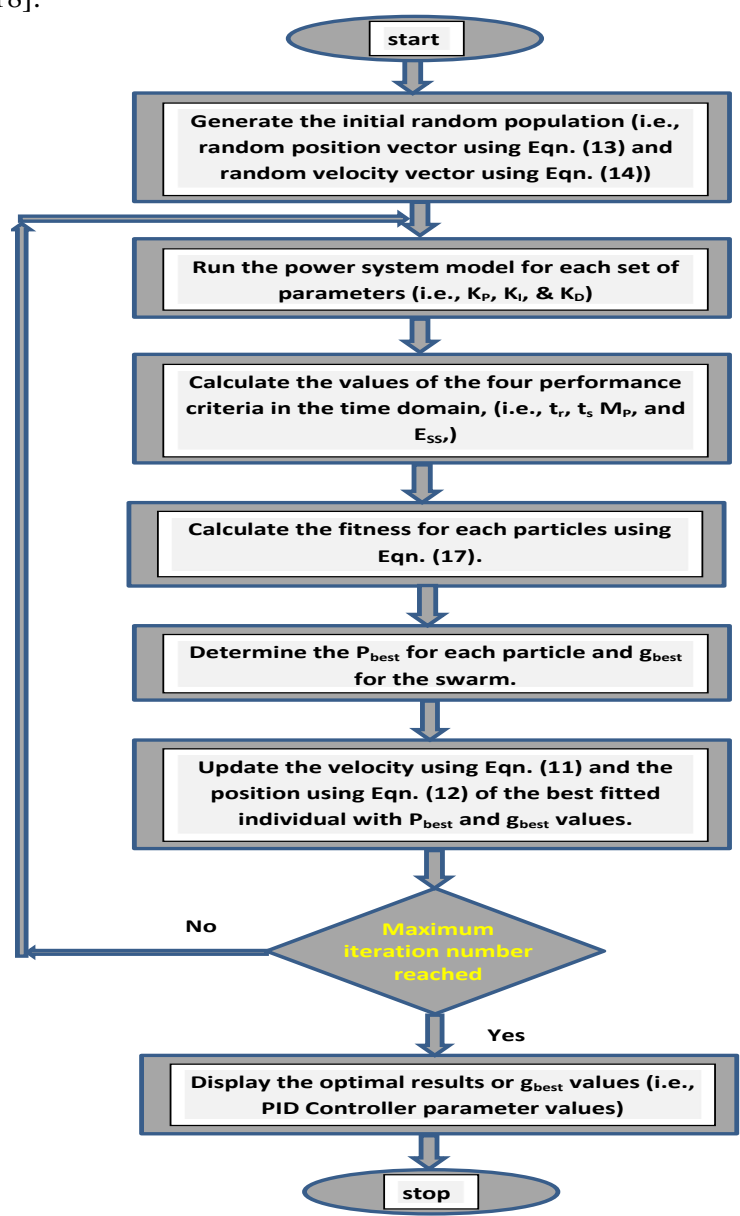

Fig 5: Flowchart of auto-tuning PSO/MPSO-PID controller

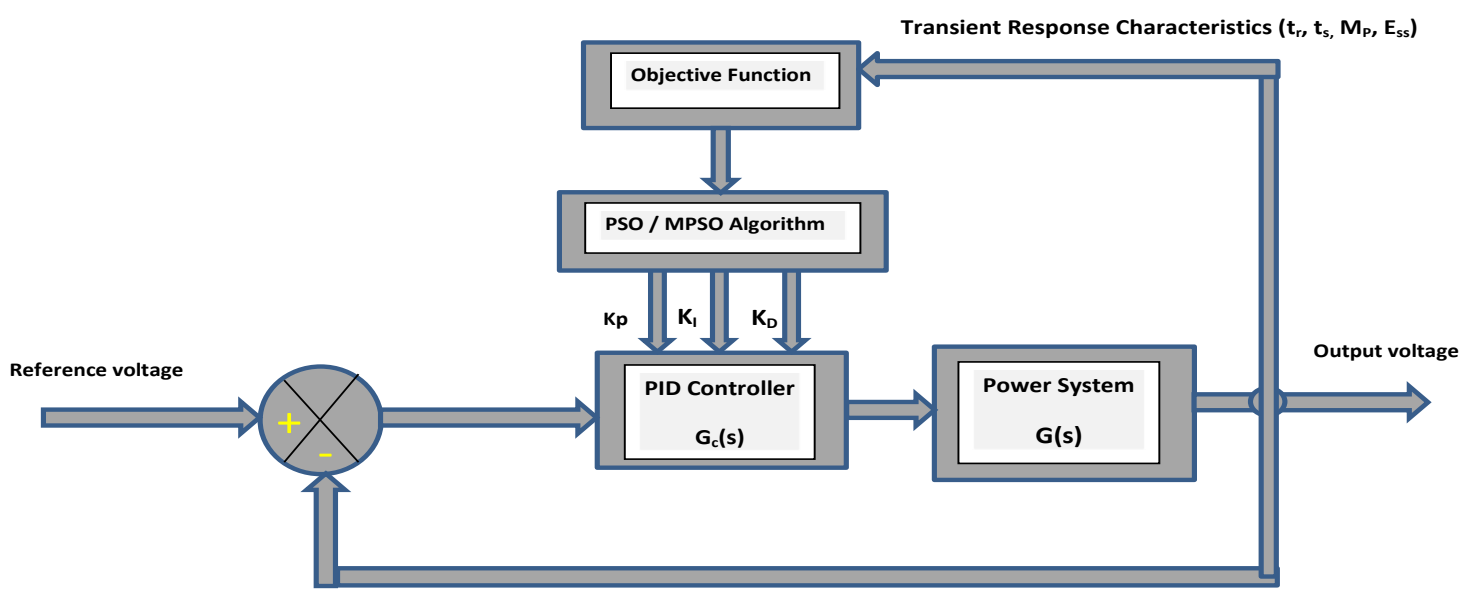

Fig 4. Block diagram of proposed PSO/MPSO-PID controller

\section{B. Fitness Function}

The most essential step in applying PSO is to choose the fitness function that is used to evaluate the fitness of each particle. In PID controller the common performance criteria are integrated absolute error (IAE), the integrated of time weight square error (ITSE) and integrated of square error (ISE) that can be evaluated analytically in the frequency domain. The three performance indices have their own advantages and disadvantages. For example, a disadvantage of the IAE and ISE indices is that its minimization can result in a response with relatively small overshoot but with long settling time. Although the ITSE performance index can overcome the disadvantage of the ISE but the calculation of this index is complex and time consuming [19]. 
In this research paper, the process of optimizing PID parameter by PSO is presented. It takes under consideration both stability and transient characteristics of the closed loop system with steady-state error serving as stability measure, while rising time, overshoot, and settling time serving as transient performance measure. Therefore, theobjective function described by Eq. (15) combined all these parameters which is minimized by PSO [19], and the fitness function has been formulated as in Eq. (15) [20].

$$
\begin{aligned}
& W(\bar{K})=\left(1-e^{-\beta}\right)\left(M_{p}+E_{s S}\right)+e^{-\beta}\left(t_{s}-t_{r}\right) \\
& J(\bar{K})=\frac{1}{W(\bar{K})}
\end{aligned}
$$

Where $\bar{K}=\left[K_{p}, K_{i}, K_{d}\right]$, and $\beta$ is the weighting factor. The fitness function can achieve the optimization requirements through setting value for $\beta$. The weighting factor can be set in the range of 0.5 to 1 in order to find out the best results for transient response characteristics and minimizing the objective function. Therefore the design problem can be formulated as:

Maximize $J(\bar{K})(17)$

Subject to

$$
\begin{aligned}
& K_{p}^{\text {min }} \leq K_{p} \leq K_{p}^{\text {max }} \\
& K_{i}^{\text {min }} \leq K_{i} \leq K_{i}^{\text {max }} \\
& K_{d}^{\text {min }} \leq K_{d} \leq K_{d}^{\text {max }}
\end{aligned}
$$

\subsection{PID Controller Tuning with Modified PSO Algorithm}

The implementation of MPSO-PID controller is achieved using same flowchart illustrated in Fig. 5 and all the previously defined equations to implement PSO-PID, the modification here is in the two more effective factors in the optimization convergence to the global optima which are the inertial weight and the acceleration factors. In this regards, a modified inertial weight has the same initial and final values as in the linear function, but is characterized by a sharp rate of decrease. A small value of $w$ encourages local exploitation whereas a larger value of $w$ support global exploration, the resultant will be improving the rate of convergence and reduced the consumed time to complete the global search. The values of $w$ are evaluated as follows [21]:

$$
w_{t}=\left(w_{\text {max }}-w_{\text {min }}\right) * \exp \left\{-\left(\frac{Z t}{t_{\text {max }}}\right)^{2}\right\}+w_{\text {min }}
$$

Where $z$ is a constant taken to be $2.2, t_{\max }$ is the maximum iteration number, $t$ is the current iteration number, $w_{\min }$ and $w_{\max }$ are the initial and final weight, respectively. In this paper, the value $w_{\min }$ and $w_{\max }$ are set to be 0.4 and 0.9 . The acceleration coefficients $c_{1}$ and $c_{2}$ are adjusted to incorporate better compromise between the exploration and exploitation of each space in PSO, time variant acceleration coefficients have been introduced in [22], and can be mathematically described as follows:

$$
\left.\begin{array}{l}
c_{1 t}=\left(c_{1 f}-c_{1 i}\right) \frac{t}{t_{\max }}+c_{1 i} \\
c_{2 t}=\left(c_{2 f}-c_{2 i}\right) \frac{t}{t_{\max }}+c_{2 i}
\end{array}\right\}
$$

where $c_{1 t}$ decreased from $c_{1 i}=2.5$ to $c_{1 f}=0.5$ and $c_{2 t}$ decreased from $c_{2 i}=0.5$ to $c_{2 f}=2.5$

\section{Results and Discussions \\ 5.1 Simulation Parameters}

The state model of the SMIB system with SVC has been derived from the state equations in section 2 , and then the transfer function of the proposed model is obtained. The simulation results are performed using MATLAB R2010a software environment run on Core(TM) i5, $2.5 \mathrm{GHz}$, and 4 GB RAM system. The simulation parameters have been taken as 25 particles, The PID controller parameters $\left(\mathrm{K}_{\mathrm{p}}, \mathrm{K}_{\mathrm{i}}\right.$ and $\left.\mathrm{K}_{\mathrm{d}}\right)$ upper and lower limits are $[2,1,1]$, weighting factor $\beta(0.5$ 0.9 ), the three loading conditions (light $\operatorname{load} P_{e}=0.4$ p.u, nominal load $P_{e}=0.8$ p.u, and heavy $\operatorname{load} P_{e}=1.2$ p.u), number of iterations (50-100). The results were rising time $\left(t_{r}\right)$, settling time $\left(t_{s}\right)$, maximum overshoot $\left(M_{p}\right)$, and steady-state error $\left(E_{S S}\right)$.

\subsection{Performance of the ZN - PID and PSO- PID Controllers}

The results of ZN-PID controller are shown in Table 2. It is clearly that the SMIB system with SVC response produces high settling time and therefore, less stability margin in comparison with the PSO-PID controller results which are demonstrated in Table 3, especially under light and nominal load conditions.

Table2.Performance of the ZN-PID controller

\begin{tabular}{|c||c|c|c|c|}
\hline \hline $\boldsymbol{P}_{\boldsymbol{e}}$ & $\boldsymbol{t}_{\boldsymbol{r}}$ & $\boldsymbol{t}_{\boldsymbol{s}}$ & $\boldsymbol{M}_{\boldsymbol{p}}$ & $\boldsymbol{E}_{\boldsymbol{s} \boldsymbol{s}}$ \\
\hline \hline 0.4 & 2 & 3.6 & 0.0224 & 0.0037 \\
\hline 0.8 & 10.7 & 19.2 & 0 & 0.0013 \\
\hline 1.2 & 32.5 & 58 & 0 & 0.0032 \\
\hline
\end{tabular}

Table 3.Performance of the PSO-PID controller

\begin{tabular}{|c|c|c|c|c|}
\hline \hline $\boldsymbol{P}_{\boldsymbol{e}}$ & $\boldsymbol{t}_{\boldsymbol{r}}$ & $\boldsymbol{t}_{\boldsymbol{s}}$ & $\boldsymbol{M}_{\boldsymbol{p}}$ & $\boldsymbol{E}_{\boldsymbol{s}}$ \\
\hline \hline 0.4 & 1.047 & 1.47 & 0.887 & $6.1 * 10^{-4}$ \\
\hline 0.8 & 5.27 & 13.23 & 0.236 & $1.73^{*} 10^{-6}$ \\
\hline 1.2 & 52.17 & 99.8 & 0 & $7.7 * 10^{-4}$ \\
\hline
\end{tabular}

\subsection{Performance of the MPSO-PID Controller}

The transient response characteristics of the MPSO-PID controller applied on SMIB system with SVC are shown in Table 4. The primary impression from the results analysis, reveal the robustness performance of the proposed tuning method and analytically, through finding the roots of the characteristic equation, it can prove the superiority of this technique, because the positions of these roots are lied in the left- hand side of S-plan and further from the imaginary axis which enhance the stability margin.

Table 4.Performance of the MPSO-PID controller

\begin{tabular}{|c|c|c|c|c|}
\hline \hline $\boldsymbol{P}_{\boldsymbol{e}}$ & $\boldsymbol{t}_{\boldsymbol{r}}$ & $\boldsymbol{t}_{\boldsymbol{s}}$ & $\boldsymbol{M}_{\boldsymbol{p}}$ & $\boldsymbol{E}_{\boldsymbol{s}}$ \\
\hline \hline 0.4 & 1 & 1.44 & 0.29 & $7.73^{*} 10^{-4}$ \\
\hline 0.8 & 2.53 & 9.58 & 0.26 & $6.74 * 10^{-6}$ \\
\hline 1.2 & 34.971 & 34.972 & 0 & 0.0023 \\
\hline
\end{tabular}

\subsection{Results Comparison of Three Proposed Controllers}

In order to show the effectiveness of the proposed MPSO tuning method, a comparison is made with PSO and ZN 
methods. Figs.6, 7 and 8 show a comparative bar graph presentation of the three tuning methods for $P_{e}=0.4,0.8$ and 1.2 respectively.

It is seen that the MPSO algorithm has a better performance in terms of maximum overshoot, which is smaller than the values obtained through PSO and $\mathrm{ZN}$ methods. In addition to this, the settling time is decreased to a very small value and is shorter than the determined value using the other two methods. It can deduce that, MPSO algorithm gets better performance than PSO and ZN methods based on transient response analysis.

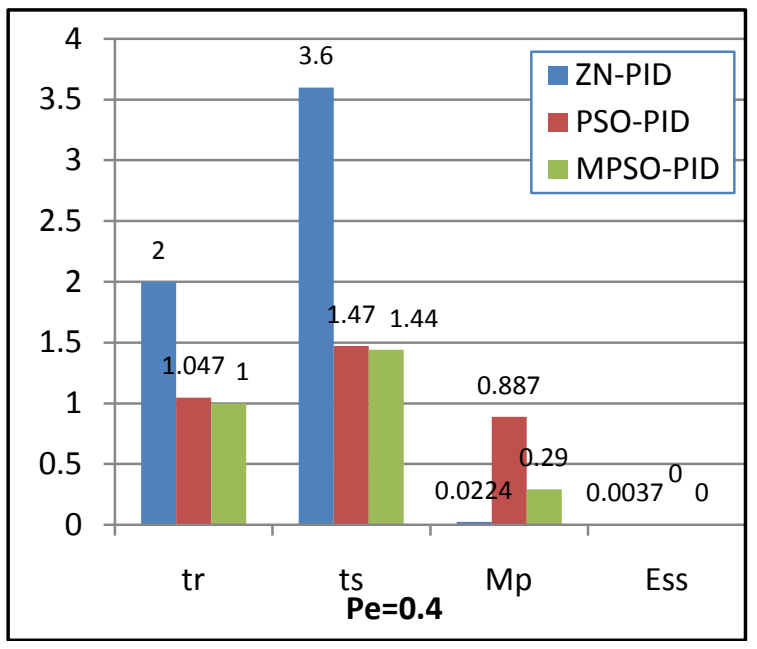

Fig 6: Performance of the proposed PID controller tuning methods at $P_{e}=0.4$

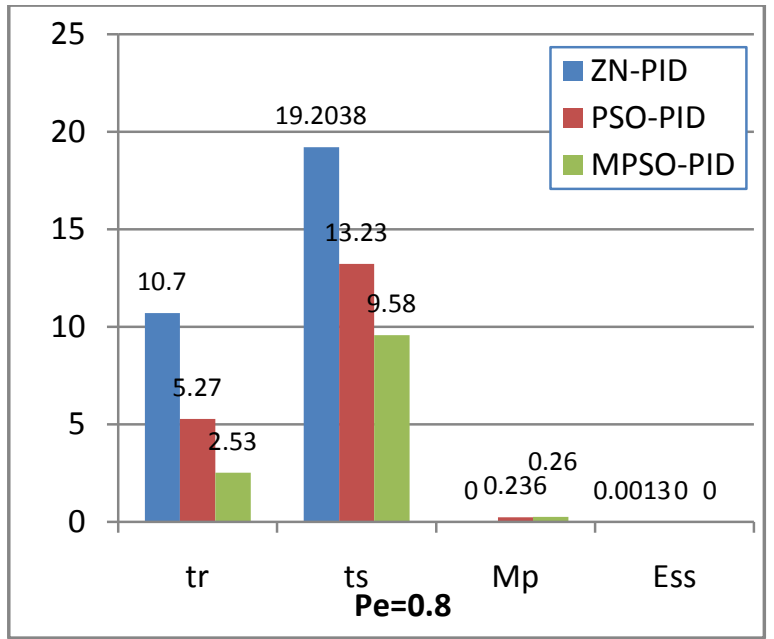

Fig 7: Performance of the proposed PID controller tuning methods at $P_{e}=0.8$

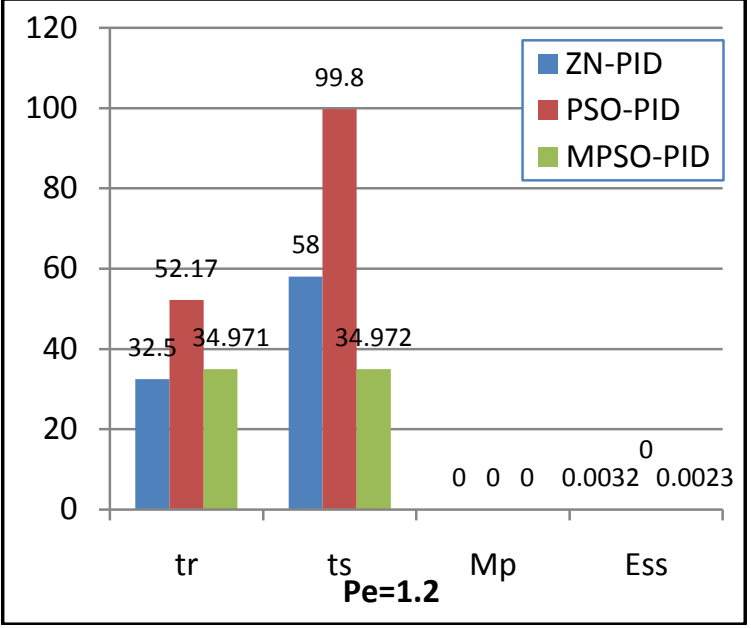

Fig 8: Performance of the proposed PID controller tuning methods at $P_{e}=1.2$

Figs. 9, 10, and 11 represent the step response of the plant based on the proposed tuning methods under different loading conditions. It can be observed that MPSO-PID controlling scheme offers superior results in terms of system performance parameters. Therefore, the behavior of the proposed MPSO tuning method shows that, the output response can follow the unexpected variations occurred in the power system efficiently.

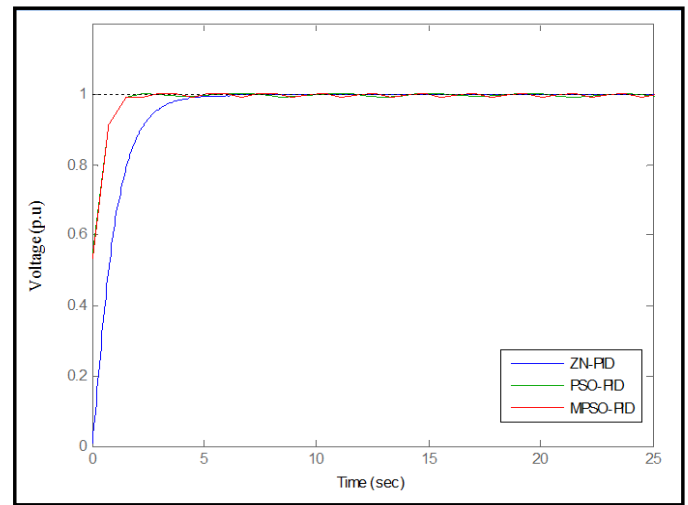

Fig 9: The response of the plant at $P_{e}=0.4$ using the three tuning methods

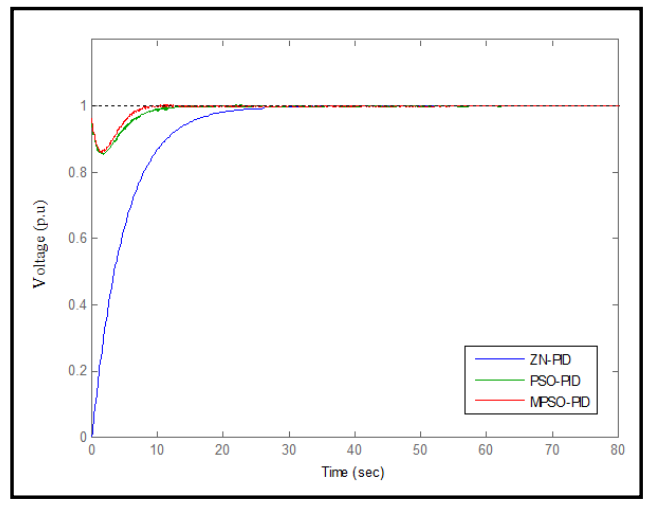

Fig 10: The response of the plant at $P_{e}=0.8$ using the three tuning methods 


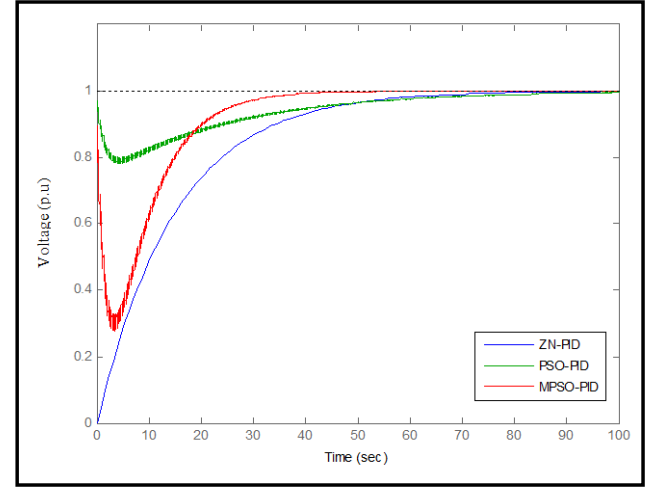

Fig 11: The response of the plant at $P_{e}=1.2$ using the three tuning methods

\section{Conclusion}

The paper presents a modified particle swarm optimization algorithm to optimize the PID controller parameters for boosting the power system stability. The studied power system is expressed by SMIB system with SVC controlled by a PID controller. The research paper problem is framed as an optimization problem in terms of PID controller parameters, and the MPSO algorithm is used to find out the optimal parameters values. An adjustable inertia weight, cognitive and social acceleration constants are used in the fitness function to guarantee the MPSO algorithm convergence. The effectiveness of the proposed technique is examined under different loading conditions, and the results are compared with the classical version of PSO and ZN tuning methods. The simulation results have shown that MPSO is able to generate optimal transient response characteristics. Therefore, the proposed strategy for power system stability enhancement combines the advantages of the PID controller tuned by MPSO and the installed SVC device to handle the load disturbance.

\section{REFERENCES}

[1] H. Saadat, "Power System Analysis," McGraw Hill International Editions, 1999.

[2] H. Rahman, Dr. F. Rahman, and H. O.-Rashid, "Stability Improvement of Power System By Using SVC With PID Controller," International Journal of Emerging Technology and Advanced Engineering (IJETAE ), vol. 2, no. 7, pp. 226-233, July 2012.

[3] H. Rahman, Dr. F. Rahman, and H. O.-Rashid, "Power System Stability Improvement By Using SVC With TID Tuned PID Controller," International Journal of Advanced Research in Computer Engineering \&Technology (IJARCET), vol. 1, no. 8, pp. 93-98, Oct. 2012.

[4] H. Rahman, Dr. F. Rahman, and H. O.-Rashid, "Improvement of Power System Stability Using Static Var Compensator with Controller," International Journal of Advanced Research in Computer Engineering \& Technology(IJARCET), vol. 1, no. 9, pp. 200-205, Nov. 2012.

[5] A. K. Mohanty, and A. K. Barik, "Power System Stability Improvement Using FACTS Devices,", International Journal of Modern Engineering Research (IJMER), vol.1, no. 2, pp-666-672, Nov.-Dec. 2011.
[6] J. G. Ziegler and N. B. Nichols, "Optimum Settings for Automatic Controllers," Transactions of ASME, vol. 64, pp. 759-765, 1942.

[7] J. Kennedy and R. Eberhart, "Particle Swarm Optimization," Proceedings of IEEE International Conference on Neural Networks, Perth, Australia, vol. IV, pp. 1942-1948, 27 Nov.-1Dec., 1995.

[8] A. E. Hammad, "Analysis of Power System Stability Enhancement by Static VAR Compensators," IEEE Transactions on Power Systems, vol. 1, no. 4, pp. 222 227, Nov. 1986.

[9] P. Srihari, and G. S. Reddy, "Effective Way to Damping Power Oscillations using Static VAR Compensator with Fuzzy Logic Controller," International Journal on Technical and Physical Problems of Engineering (IJTPE), vol. 4, no. 13, pp. 89-94, Dec. 2012.

[10] Y. Ma, S. Chen, and B. Zhang, "A Study on Nonlinear SVC Control for Improving Power System Stability," Proceedings of IEEE Region 10 Conference on Computer, Communication, Control and Power Engineering (TENCON'93), Beijing, China, vol. 5, pp. 166-169, 19-21 Oct. 1993.

[11]R. V. Dukkipati, "Control Systems," Narosa Publishing House, 2005.

[12] K. Ang, G. Chong, and Y. Li, "PID Control System Analysis, Design, and Technology," IEEE Trans. Control System Technology, vol. 13, pp. 559-576, July 2005.

[13]K. S. Ismail, F. M. Tuaimah, and R. A. Hummadi, "DC Motor Speed Controller Design using Pole Assignment Technique for Industrial Application, "Journal of Engineering, vol. 17, no. 3,pp. 466-472, June 2011.

[14] B. S. Manke, "Linear Control Systems with MATLAB Applications," Khanna Publishers, $8^{\text {th }}$ Edition, 2006.

[15] K. J. Åström and R. M. Murray, "Feedback Systems: An Introduction for Scientists and Engineers," Princeton University Press, Princeton, NJ, 2008.

[16]K. J. Åström and T. Hägglund, "PID Controllers: Theory, Design, and Tuning," Instrument Society of America, Research Triangle Park, NC, $2^{\text {nd }}$ Edition, 1995.

[17] K. E. Parsopoulos, M. N. Vrahatis, "Particle Swarm Optimization and Intelligence: Advances and Applications," Information Science Reference, NY, USA, 2010

[18] M. Clerc, "The swarm and the Queen: Towards a Deterministic and Adaptive Particle Swarm Optimization, "Proceedings of International Conference on Evolutionary Computation, France Telecom, Annecy, France, vol. 3, pp. 1951-1957, 06-09 July, 1999.

[19]Z.-L. Gaing, "A Particle Swarm Optimization Approach for Optimum Design of PID Controller in AVR System," IEEE Transactions on Energy Conversion, vol. 19, no. 2,pp. 384-391, June 2004.

[20]T. O. Mahony, C. J. Downing, and K.Fatla, "Genetic Algorithm for PID Parameter Optimization: Minimizing Error Criteria," Proceedings of International Conference on Process Control and Instrumentation, Glasgow, UK, pp. 148-153, 26-28 July, 2000. 
[21] M. A. Zaman , S. A. Mamun, Md. Gaffar, S. M.Choudhury, Md. M. Alam, and Md. Abdul Matin, "Phased Array Synthesis Using Modified Particle Swarm Optimization," Journal of Engineering Science and Technology Review, vol. 4, no. 1, pp. 68-73, 2011.
[22] A. Ratnaweera, S. K. Halgamuge, and H. C. Watson, "Self-Organizing Hierarchical Particle Swarm Optimizer with Time-Varying Acceleration coefficients," IEEE Transactions on Evolutionary Computation, vol. 8, no. 3, pp. 240-255, 2004. 